\title{
Wie gross sollen Altholzinseln sein?
}

\author{
Markus Müller \\ Thibault Lachat \\ Rita Bütler
}

Eidgenössische Forschungsanstalt für Wald, Schnee und Landschaft (CH)

Eidgenössische Forschungsanstalt für Wald, Schnee und Landschaft $(\mathrm{CH})^{*}$

Eidgenössische Forschungsanstalt für Wald, Schnee und Landschaft $(\mathrm{CH})$

\section{How large should old-growth islands be?}

\begin{abstract}
About $5 \%$ of the Swiss forest surface should be protected as natural forest reserves or as old-growth islands until 2030. This goal, which has been formulated by the Federal Office for the Environment and by the cantons, aims to promote the natural development of the forest and to conserve saproxylic species. Beside large-sized natural forest reserves, smaller old-growth islands may also play an important role. A bootstrapping method has been developed to define the minimal size of old-growth islands based on a field inventory in high forests without logging since at least 30 years. Three different indicators for habitat structures have been used to establish the ecological potential of old-growth islands: the total number of habitat structures, the amount of deadwood and the number of woodpecker trees. The threshold has been defined as the average of each indicator minus the standard error for different forest types. The surface of an island is determined at the point where $75 \%$ of the modeled surfaces are above the threshold, which guarantees a certain ecological potential. This surface reaches 0.7 ha for floodplain forests, 0.9 ha for beech and fir-beech forests and 1.2 ha for silver spruce-fir forests. The computed values are minimal rather than optimal surfaces for old-growth islands. With an increase of the surface, sustainability, quantity and quality of the habitats will be improved. Therefore, the size of old-growth islands should be larger whenever the local circumstances are favorable.
\end{abstract}

Keywords: saproxylic species, deadwood, veteran trees, old-growth island, habitat structures doi: 10.3188.szf.2012.0049

* Zürcherstrasse 111, CH-8903 Birmensdorf, E-Mail thibault.lachat@wsl.ch

$\mathrm{D}$ ie Förderung von Alt- und Totholz ist ein wesentlicher Bestandteil der Strategie des Bundesamtes für Umwelt zur Erhaltung der Biodiversität im Wald. Die Tatsache, dass rund 20 bis $25 \%$ der waldbewohnenden Arten auf Totholz angewiesen sind, verdeutlicht, wie wichtig diese Ressource für die Waldbiodiversität ist (z.B. Elton 1966, Siitonen 2001). Obwohl das durchschnittliche Totholzvolumen im Schweizer Wald in den letzten 20 Jahren von $11.9 \mathrm{~m}^{3} / \mathrm{ha}$ auf $18.5 \mathrm{~m}^{3} /$ ha zugenommen hat (Brändli \& Abegg 2009), bleibt die Situation für xylobionte (holzbewohnende) Arten in gut zugänglichen Wäldern weiterhin kritisch. Vor allem im Jura und im Mittelland werden die vorgeschlagenen Totholzvorräte von 20 bis $40 \mathrm{~m}^{3} /$ ha selten erreicht (Bütler \& Lachat 2009). Lediglich im Bereich der Voralpen und Alpen sind solche Werte häufiger (Brändli \& Abegg 2009). Somit ist erklärt, weshalb vor allem im Jura und im Mittelland weitere Massnahmen zur Alt- und Totholzförderung nötig sind.
Grosse Waldbestände ohne Bewirtschaftung sind aus Sicht des Naturschutzes sehr wünschenswert, aus Sicht der Holzproduktion sollte der Ertragsausfall jedoch nicht unverhältnismässig gross sein. Zudem ist eine nachhaltige Nutzung des Rohstoffes Holz auch im Sinne des Natur- und Umweltschutzes. Durch den langfristig wahrscheinlich weiter zunehmenden Bewirtschaftungsdruck (v.a. zunehmende Nachfrage nach Energieholz) wird die Ausweisung von grossflächigen Naturwaldreservaten in gut erschlossenen Wäldern des Juras und des Mittellandes oft auf Widerstand stossen. Altholzinseln stellen eine nützliche Ergänzung des Biotopverbundes zu den meist weit voneinander entfernten Waldreservaten dar. Sie dienen gleichzeitig als Habitat und Trittstein für die Ausbreitung xylobionter Arten (z.B. Irmler et al 2010). Als Altholzinseln werden kleinflächige Bereiche im Wirtschaftswald bezeichnet, welche nicht genutzt werden und durch ihren hohen Anteil an alten Bäumen und Totholz wertvolle 


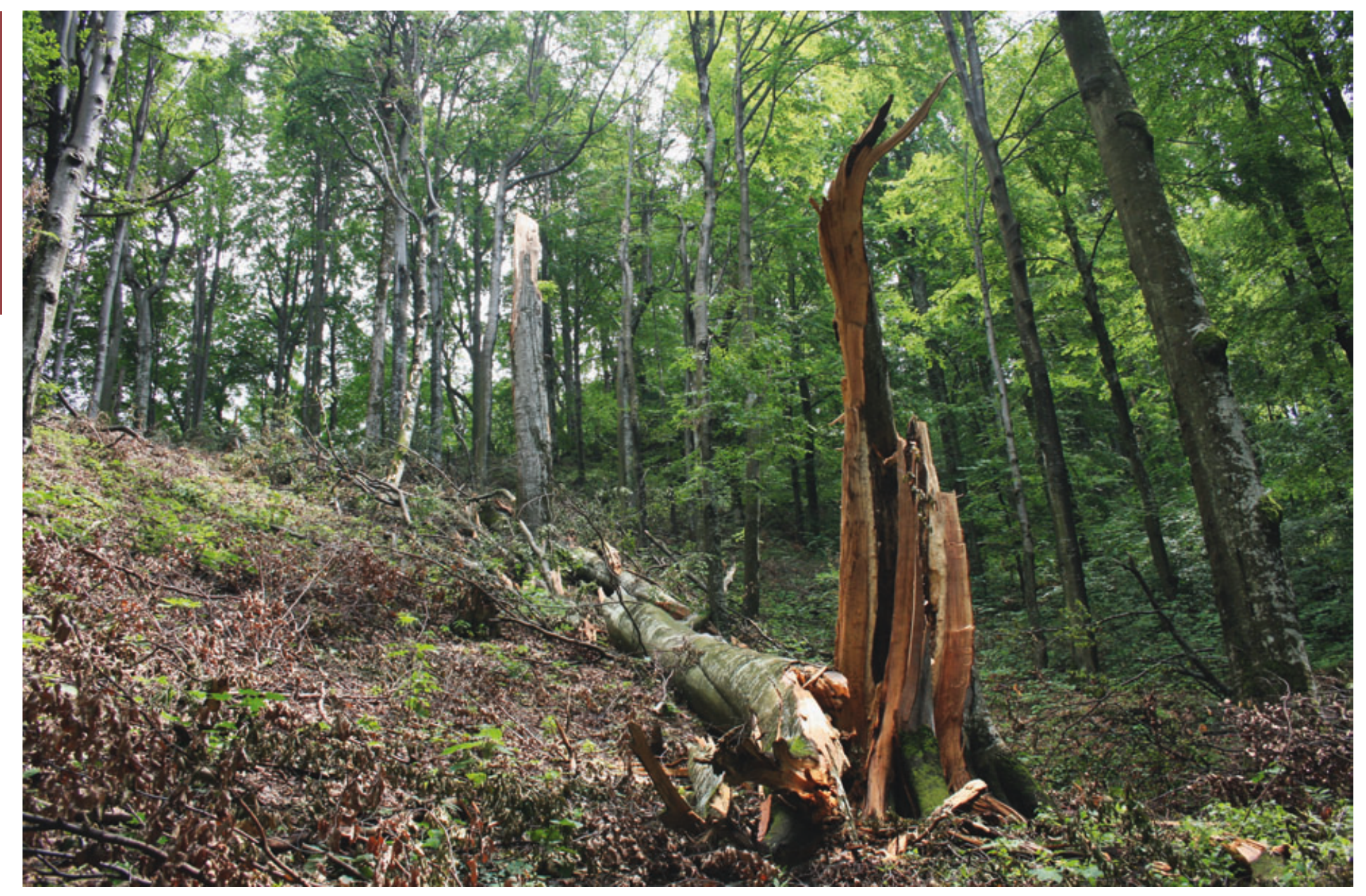

Abb 1 In Altholzinseln kann sich eine natürliche Dynamik des Zerfalls einstellen.
Lebensräume für verschiedenste Organismen sind. So kann sich zumindest kleinräumig eine natürliche Dynamik des Zerfalls einstellen (Abbildung 1).

Damit Altholzinselprojekte zielführend umgesetzt werden, ist es wichtig, Minimalflächen für Altholzinseln zu definieren. So kann sichergestellt werden, dass die ausgeschiedenen Flächen einen grossen Wert für die Biodiversität erreichen und dennoch die Waldnutzung möglichst wenig einschränken.

Die Grösse eines Schutzgebietes kann anhand von Artenansprüchen bestimmt werden. Dafür werden Schirm- respektive Indikatorarten betrachtet, deren Schutz das Überleben einer ganzen Lebensgemeinschaft sichern soll. Dieser artenspezifische Ansatz wird jedoch kontrovers diskutiert (z.B. Seddon \& Leech 2008, Roberge \& Angelstam 2004). Andelman \& Fagan (2000) kommen in ihrer Untersuchung gar zum Schluss, dass mit gezielt ausgewählten Schirm- oder Indikatorarten sich die Grösse eines Schutzgebietes nicht besser bestimmen lässt als mit zufällig ausgewählten Arten. Zudem sind für diesen Ansatz umfassende Kenntnisse der ausgewählten Arten nötig. Diese fehlen bei sehr vielen xylobionten Arten insbesondere hinsichtlich ihrer Raumansprüche.

Für Lindenmayer et al (2000) sind strukturbasierte Indikatoren zur Beurteilung der Biodiversität in Wirtschaftswäldern eine gute Alternative zu einem artenbasierten Ansatz. Winter (2008) und Winter \& Möller (2008) belegen zudem, dass die xylobionte Vielfalt in Wäldern sehr gut anhand von Habitatstrukturen beurteilt werden kann. Habitatstrukturen sind morphologische Besonderheiten eines Baums, die für eine oder mehrere Alt- oder Totholzarten ein bevorzugtes Habitat darstellen, also zum Beispiel eine Baumhöhle, eine Rindenverletzung oder ein toter Ast (Bütler \& Lachat 2009; Abbildung 2). Anhand dieser Habitatstrukturen kann das ökologische Potenzial einer Altholzinsel gemessen werden. Dabei spielt nicht nur die Anzahl der Habitatstrukturen eine Rolle, sondern auch ihre Vielfalt (Bütler \& Lachat 2009). Grundsätzlich gilt, dass das ökologische Potenzial einer Altholzinsel umso grösser ist, je mehr Habitatstrukturen sie aufweist.

Wälder, die seit 30 Jahren nicht mehr bewirtschaftet werden, können bereits erhebliche Mengen an Totholz und viele Habitatstrukturen aufweisen (Meyer \& Schmidt 2011). Bütler \& Lachat (2009) konnten zeigen, dass solche Wälder ähnliche Totholzmengen wie Natur- und sogar Urwälder in Europa bergen und die Anzahl der aufgenommenen Habitatstrukturen in etwa den Beobachtungen von Winter \& Möller (2008) in 350-jährigen, seit über 100 Jahren nicht mehr bewirtschafteten Buchenwäldern entspricht.

Das Ziel dieser Studie ist es, die Minimalfläche für Altholzinseln in verschiedenen Waldtypen 
zu bestimmen, sodass deren Ausstattung punkto Totholz und Habitatstrukturen bestimmten ökologischen Anforderungen entspricht.

\section{Methode}

\section{Datengrundlage}

Um die Minimalflächen für Altholzinseln möglichst differenziert definieren zu können, wurden vier Waldtypen in neun verschiedenen Kantonen untersucht (Tabelle 1): Auenwald, Buchenwald, Tannen-Buchen-Wald und Tannen-Fichten-Wald. An jedem Standort wurden vier Stichproben à $500 \mathrm{~m}^{2}$ in besonders altholzreichen, mindestens 30 Jahre nicht bewirtschafteten Hochwäldern aufgenommen (für Details zu den Waldtypen und den untersuchten Waldbeständen siehe Bütler \& Lachat 2009).

Auf jeder Probefläche wurden alle Habitatstrukturen erfasst (z.B. tote und gebrochene Äste, Bruthöhlen, Mulmhöhlen, Risse, Rindenverletzun- zählige Organismen von ihnen profitieren (Winter 2008), und Spechte sind gute Indikatoren für die Vielfalt der Vogelwelt sowie für den Zustand und die Baumartenvielfalt von Wäldern (z.B. Remm et al 2006, Drever et al 2008). Der Parameter «Totholz» wird seit Längerem auf internationaler Ebene als Indikator der Biodiversität in Waldökosystemen benutzt (MCPFE 2003). Die Kombination der drei Parameter repräsentiert die Vielfalt des Lebensraumes und diente in dieser Untersuchung zur Beurteilung der ökologischen Qualität einer Altholzinsel.

\section{Bestimmung der Schwellenwerte}

Wälder in der Zerfallsphase weisen meistens eine heterogene Struktur auf. Durch einen absterbenden oder umstürzenden Baum entsteht kleinräumig eine Anhäufung von Totholz, lichtreichen Habitaten und zusätzlichen Habitatstrukturen, während auf direkt angrenzenden Flächen diese Strukturen nahezu fehlen können. Unser Ziel war es, diejenige Flächengrösse zu bestimmen, bei der eine Altholz-
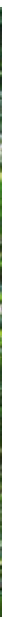

Abb 2 Totholz, Spechtbäume und Habitatstrukturen (hier eine Rindenverletzung) wurden zur Modellierung der Minimalfläche von Altholzinseln verwendet.

gen, Konsolenpilze; Bütler \& Lachat 2009). Daraus ergaben sich einerseits die Anzahl Habitatstrukturen und andererseits die Anzahl Spechtbäume. Als Spechtbaum wurde ein Baum bezeichnet, der mindestens eine Spechthöhle aufwies. Zusätzlich wurde das liegende und stehende Totholzvolumen quantifiziert. Das liegende Totholz wurde ab einem Durchmesser von $7 \mathrm{~cm}$ gemäss der Transektmethode des Schweizerischen Landesforstinventars (Böhl \& Brändli 2007) aufgenommen, das stehende ab einem Brusthöhendurchmesser (BHD) von $10 \mathrm{~cm}$. Sein Volumen wurde mit der Prozedur von Kaufmann (2000) hergeleitet.

Die drei Parameter «Anzahl Habitatstrukturen», «Anzahl Spechtbäume» und «Totholzmenge (stehend und liegend)» wurden für die Modellierung der Minimalfläche verwendet. Habitatstrukturen sind typisch für Waldbestände in späten Entwicklungsstadien (Bütler \& Lachat 2009), Spechthöhlen stellen eine wichtige Habitatstruktur dar, indem un- insel eine minimale Anzahl Habitatstrukturen, Spechtbäume oder eine minimale Totholzmenge pro Fläche aufweist, d.h., einen geforderten Schwellenwert für diese Parameter übertrifft.

Definiert wurde dieser Schwellenwert als Mittelwert abzüglich des Standardfehlers für jede der drei erfassten Grössen. Dieses Vorgehen wird durch drei Tatsachen gerechtfertigt:

- Die im Rahmen dieser Studie untersuchten Bestände haben ähnliche Mengen Totholz und Habitatstrukturen wie Natur- und Urwälder Europas. Sie werden in dieser Studie als Referenzen für erreichbare Ziele verwendet (Bütler \& Lachat 2009).

- Die natürliche Heterogenität des Waldes und damit die zum Teil grosse Variabilität der Daten wird durch diesen im Vergleich zum absoluten Mittelwert geringeren Wert berücksichtigt.

- Die Qualität einer Altholzinsel erhöht sich im Lauf der Zeit, indem sich bis zur Zerfallsphase immer mehr Strukturen ausbilden. 


\begin{tabular}{|c|c|c|c|c|c|}
\hline \multirow[t]{2}{*}{ Waldtyp } & \multirow[t]{2}{*}{ Parameter } & \multirow[t]{2}{*}{ Mittelwert } & \multirow[t]{2}{*}{ Standardfehler } & \multicolumn{2}{|c|}{ Schwellenwert } \\
\hline & & & & Absolut & $\%$ des Mittelwertes \\
\hline \multirow[t]{3}{*}{ Auenwald } & Totholz & 118.4 & 23.3 & 95.1 & 80.3 \\
\hline & Habitatstrukturen & 12.1 & 2.04 & 10.06 & 83.1 \\
\hline & Spechtbäume & 0.4 & 0.16 & 0.24 & 60 \\
\hline \multirow[t]{3}{*}{ Buchenwald } & Totholz & 120.7 & 21.0 & 99.3 & 82.2 \\
\hline & Habitatstrukturen & 13.29 & 0.99 & 12.3 & 92.5 \\
\hline & Spechtbäume & 0.29 & 0.11 & 0.18 & 62 \\
\hline \multirow[t]{3}{*}{ Tannen-Buchen-Wald } & Totholz & 143.1 & 25.1 & 118 & 82.5 \\
\hline & Habitatstrukturen & 9.29 & 1.1 & 8.19 & 88.1 \\
\hline & Spechtbäume & 0.17 & 0.08 & 0.09 & 53 \\
\hline \multirow[t]{3}{*}{ Tannen-Fichten-Wald } & Totholz & 98.1 & 18.3 & 79.8 & 81.3 \\
\hline & Habitatstrukturen & 9.79 & 0.89 & 8.7 & 88.9 \\
\hline & Spechtbäume & 0.21 & 0.08 & 0.13 & 62 \\
\hline
\end{tabular}

Tab 1 Schwellenwerte (geforderte Minimalwerte) für die einzelnen Parameter, gruppiert nach Waldtypen. Die Werte des Totholzes (stehend und liegend) sind in $\mathrm{m}^{3} /$ ha angegeben, diejenigen für die Habitatstrukturen und Spechtbäume in Stück pro Probefläche (0.05 ha). Zugrunde liegende Anzahl Bestände (Stichproben): Auenwald 5 (20), Buchenwald 6 (24), Tannen-Buchen-Wald 6 (24), Tannen-Fichten-Wald 7 (28).

\section{Modellierung der Minimalfläche}

Ausgehend von den Felddaten wurden für die vier verschiedenen Waldtypen diejenigen minimalen Flächengrössen bestimmt, bei welchen Altholzinseln mit grosser Wahrscheinlichkeit eine vordefinierte Anzahl an Habitatstrukturen und Spechtbäumen sowie eine vordefinierte Menge an Totholz erreichen. Dazu wurden die im Feld erhobenen Stichproben zufällig miteinander kombiniert, um Reihen neuer, grossflächigerer Stichproben zu modellieren. Dieses Verfahren des Resamplings wird bei ökologischen Fragestellungen oft angewendet (z.B. Ricotta 2009, Denny et al 2009). Es erlaubt durch die zufällige Kombination, die natürliche Vielfalt des Waldes abzubilden.

Durch die Kombination von zwei 0.05 ha grossen Stichproben erzeugt man eine 0.1 ha grosse, modellierte Stichprobe, durch die Kombination von drei Stichproben eine 0.15 ha grosse usw. Durch dieses Verfahren kann eine Reihe von Flächen in 0.05-ha-Schritten modelliert werden, wobei die maximal modellierbare Flächengrösse der Gesamtfläche aller reellen Stichproben entspricht. Diese Beschränkung hatte aber keinen Einfluss auf die Ergebnisse, da die Schwellenwerte immer bereits bei einer kleineren Fläche erreicht wurden als der maximal modellierbaren Flächengrösse.

\begin{tabular}{|l|c|c|c|c|}
\hline & $\begin{array}{c}\text { Auenwald } \\
\text { (ha) }\end{array}$ & $\begin{array}{c}\text { Buchenwald } \\
\text { (ha) }\end{array}$ & $\begin{array}{c}\text { Tannen- } \\
\text { Buchen-Wald } \\
\text { (ha) }\end{array}$ & $\begin{array}{c}\text { Tannen- } \\
\text { Fichten-Wald } \\
\text { (ha) }\end{array}$ \\
\hline Totholz & 0.6 & 0.6 & $\mathbf{0 . 9}$ & 0.7 \\
\hline Habitatstrukturen & 0.6 & 0.6 & 0.7 & 0.9 \\
\hline Spechtbäume & $\mathbf{0 . 7}$ & $\mathbf{0 . 9}$ & 0.8 & $\mathbf{1 . 2}$ \\
\hline
\end{tabular}

Tab 2 Minimale Fläche für Altholzinseln, die die festgelegten Schwellenwerte für die Parameter Totholzvolumen, Anzahl Habitatstrukturen und Anzahl Spechtbäume mit einer Wahrscheinlichkeit von $\geq 75 \%$ übertreffen. Die für die Bestimmung der Minimalfläche im jeweiligen Waldtyp massgebliche Grösse ist fett markiert.
In einem ersten Schritt wurden für jede zu modellierende Flächengrösse 10000 zufällige Stichproben erzeugt (Bootstrapping) und deren Anteil bestimmt, welcher die geforderten Schwellenwerte übertrifft. Dieser Vorgang (Modellierung und Vergleich mit Schwellenwert) wurde 100-mal wiederholt. Der Mittelwert dieser 100 Anteile wurde für die Bestimmung der Minimalflächen verwendet (Abbildung 3). Die Minimalfläche einer Altholzinsel wurde abschliessend definiert als diejenige Grösse, bei der $75 \%$ der Flächen den Schwellenwert übertreffen, sodass das ökologische Potenzial mit hoher Wahrscheinlichkeit (75\%) erreicht wird. Um sicherzustellen, dass von allen drei Parametern (Habitatstrukturen, Spechtbäume und Totholz) $75 \%$ der Flächen die Schwellenwerte erreichen, wird pro Waldtyp der grösste Wert der drei Parameter als Minimalfläche vorgeschlagen.

\section{Resultate}

Die anhand des Bootstrapping-Modells bestimmte minimale Flächengrösse für Altholzinseln bewegt sich in einem Bereich von 0.7 ha (Auenwald) bis 1.2 ha (Tannen-Fichten-Wald; Tabelle 2). Alle Datensätze, aus denen die Minimalflächen abgeleitet wurden, zeigen eine ähnliche Entwicklung (Abbildung 3): Zu Beginn nimmt das ökologische Potenzial einer Altholzinsel mit der Vergrösserung ihrer Fläche rasch zu. Diese anfänglich starke Zunahme flacht sich bei allen drei betrachteten Parametern ab und tendiert allmählich gegen null. Beim Parameter «Spechtbäume» wurden Schwankungen im Anteil geeigneter Flächen festgestellt, die durch die geringe Anzahl an Spechtbäumen auf den Stichprobenflächen von 0.05 ha bedingt sind. Deshalb nimmt mit zunehmender Flächengrösse der Anteil geeigneter 


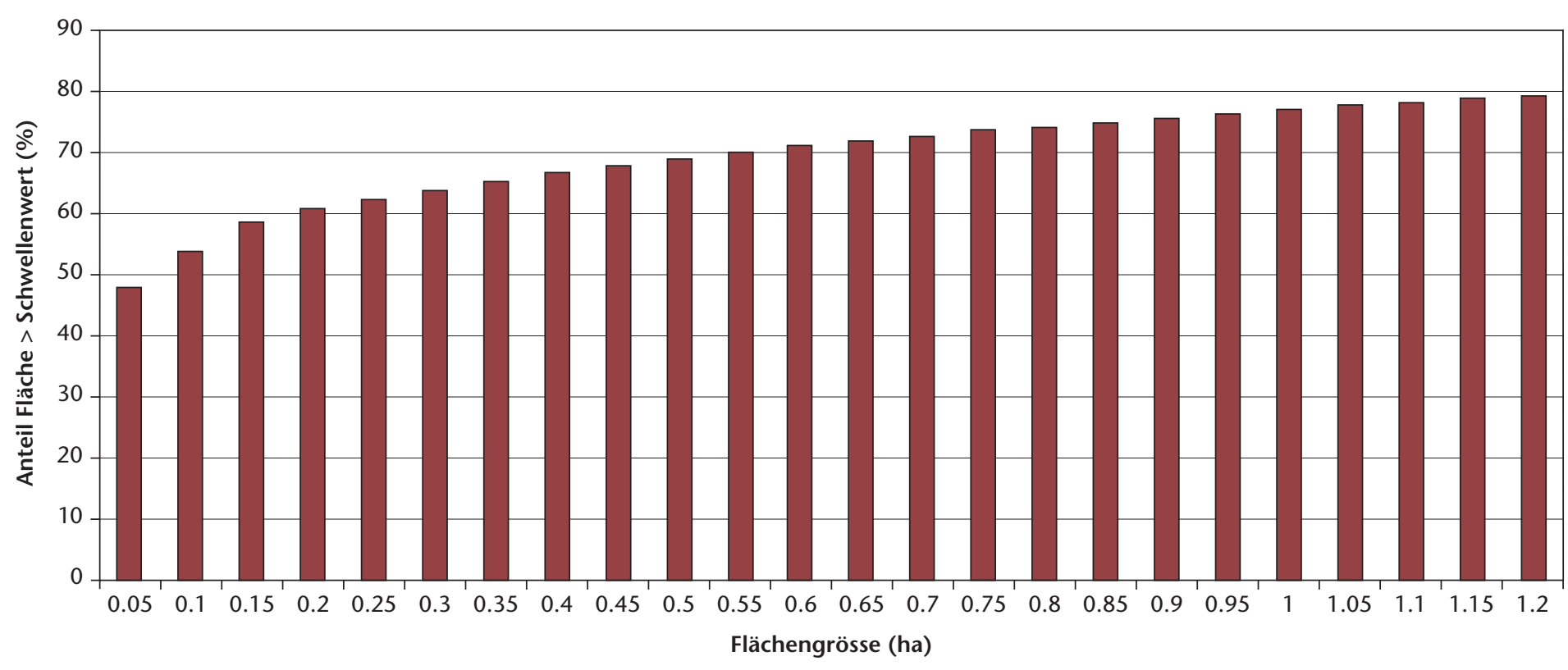

Abb 3 Modellierter Datensatz für das Totholzvolumen im Tannen-Buchen-Wald. Die Höhe der Säulen stellt den Mittelwert des Prozentsatzes an Flächen dar, die den Schwellenwert übertreffen.

Flächen nicht stetig zu. Je grösser die modellierten Flächen sind, desto kleiner werden diese Schwankungen. Dies hat zur Folge, dass für die Spechtbäume nicht diejenige Fläche als Minimalfläche bestimmt wird, bei welcher der Wert von 75\% das erste Mal erreicht, sondern diejenige, ab welcher der Wert von $75 \%$ nicht mehr unterschritten wird.

\section{Diskussion}

\section{Grösse einer Altholzinsel}

Im Bereich, in welchem 75\% der Flächen den Schwellenwert übertreffen, ist die Zunahme des ökologischen Potenzials nur noch schwach. Dennoch ist es wichtig, dass die geforderten Minimalflächen auch als solche verstanden und nicht als optimale Grösse von Altholzinseln interpretiert werden. Wenn die Gegebenheiten es zulassen, ist das Ausscheiden von grösseren Altholzinseln zu begrüssen, da eine grosse Altholzinsel neben der grösseren Wahrscheinlichkeit für eine hohe Dichte an Habitatstrukturen, Totholz und Spechtbäumen weitere Vorteile hat (z.B. Arten-Areal-Beziehung; Arrhenius 1920). Die stete Zufuhr von alten Bäumen und Totholz zur Aufrechterhaltung der Funktion einer Altholzinsel (vgl. Grove 2002, Jedicke 2008) ist in einer grossen Altholzinsel auf eine längere Zeit gesichert als in einer kleinen. In sehr kleinen Altholzinseln besteht insbesondere das Risiko, dass der gesamte Bestand in kurzer Zeit zusammenbricht und sich auf der ganzen Fläche Jungwald etabliert. Mit zunehmender Grösse einer Altholzinsel steigt auch die Chance, dass ein Kontinuum an Alt- und Totholz über längere Zeit aufrechterhalten bleibt.

Jakoby et al (2010) sind aufgrund der Modellierung der Totholzdynamik in Buchenbeständen zum Schluss gekommen, dass es eine Fläche von mindestens 0.5 ha braucht, damit immer etwas stehendes und liegendes Totholz in einer Altholzinsel vorhanden ist. In den im Rahmen unserer Studie untersuchten Buchenbeständen wurden im Durchschnitt $120 \mathrm{~m}^{3} /$ ha Totholz gemessen. Nach Jakoby et al (2010) ist eine Fläche von 0.73 ha notwendig, um diese Totholzmenge im Durchschnitt langfristig zu gewährleisten. Um unseren Schwellenwert von $99.3 \mathrm{~m}^{3}$ /ha Totholz im Buchenwald zu erreichen (Tabelle 1), bräuchte es nach unseren Berechnungen eine Fläche von 0.6 ha, was den Ergebnissen von Jakoby et al (2010) entspricht (0.5 ha für $84 \mathrm{~m}^{3} /$ ha im Durchschnitt).

Der Abschluss von Verträgen mit langer Laufzeit ermöglicht es, den administrativen Aufwand für die Sicherung von Altholzinseln klein zu halten. Damit langfristige Verträge aber Sinn machen, muss über eine entsprechende Minimalfläche sichergestellt werden, dass die Altholzinsel ihre Funktion über die gesamte Laufzeit erfüllen kann. Werden Verträge mit kürzeren Laufzeiten abgeschlossen, so müssen nach deren Ablauf die Altholzinseln erneut beurteilt und es muss nach Ersatz gesucht werden, falls sie nicht mehr den Anforderungen entsprechen.

Eine langfristig bestehende Altholzinsel hat zudem ökologische Vorteile. Wie Grove (2002) ausführt, ist das Fehlen eines kontinuierlichen Angebots an Totholz in vielen europäischen Wäldern vermutlich eine der Hauptursachen für das Verschwinden vieler xylobionter Arten. Dementsprechend ist es wichtig, Altholzinseln auszuscheiden, die über einen langen Zeitraum bestehen, damit sich gefährdete Arten über lange Zeit am selben Ort entwickeln können. Zudem sollte bei der Wahl von Ersatzstandorten für nicht mehr funktionsfähige Altholzinseln darauf geachtet werden, dass die neuen Flächen mög- 
lichst nahe bei den zu ersetzenden zu liegen kommen, um die Migration der Organismen zu ermöglichen.

\section{Vergleich mit Naturwäldern}

Mit Altholzinseln und Naturwaldreservaten sollen die im Schweizer Wirtschaftswald fast gänzlich fehlenden Zerfallsphasen gefördert werden. Charakteristisch für diese Zerfallsphasen ist eine grosse Totholzmenge. Eine Gegenüberstellung der verwendeten Schwellenwerte für das Totholzvolumen (Tabelle 1) mit Werten von Naturwaldreservaten zeigt auf, dass diese durchaus vergleichbar sind. So fanden Christensen et al (2005) für europäische, seit mehr als 50 Jahren bestehende Buchenwaldreservate ein durchschnittliches Totholzvolumen von $132 \mathrm{~m}^{3} / \mathrm{ha}$. Siitonen et al (2000) wiesen in Fichtenwaldreservaten in Finnland eine durchschnittliche Totholzmenge von $111 \mathrm{~m}^{3} / \mathrm{ha}$ nach. Somit stellen Altholzinseln zumindest kleinräumig Totholzmengen bereit, wie sie auch in Naturwaldreservaten vorgefunden werden.

Im Rahmen der Waldreservatspolitik Schweiz wurde das Ziel definiert, bis 2030 etwa 5\% der Schweizer Waldfläche als Naturwaldreservate und Altholzinseln auszuweisen. ${ }^{1}$ Im Vergleich dazu befinden sich in Urwäldern Europas zwischen 20 und 58\% der Waldfläche in der Zerfallsphase (Tabelle 3).

\begin{tabular}{|l|l|c|l|}
\hline $\begin{array}{l}\text { Geografische } \\
\text { Lage }\end{array}$ & Waldtyp & $\begin{array}{c}\text { Anteil Zerfallsphase } \\
(\%)\end{array}$ & Quelle \\
\hline Karpaten & Buchenwald & $42-45$ & Korpel (1995) \\
\hline Albanien & Buchenwald & $48-57$ & Tabaku (1999) \\
\hline Osteuropa & Tannen-Buchen-Wald & $20-25$ & Leibundgut (1982) \\
\hline Karpaten & Tannen-Buchen-Wald & 35 & Korpel (1995) \\
\hline $\begin{array}{l}\text { Osteuropa/ } \\
\text { Deutschland }\end{array}$ & Tannen-Fichten- & $32-58$ & $\begin{array}{l}\text { Mayer \& Neumann } \\
\text { Buchen-Wald }\end{array}$ \\
\hline
\end{tabular}

Tab 3 Übersicht über den Anteil der Zerfallsphase in verschiedenen Urwäldern Europas. Bei den Untersuchungen von Mayer \& Neumann (1981) sowie denen von Tabaku (1999) wurden die Werte der Zerfallsphase und der Terminalphase addiert, da dies in etwa der Zerfallsphase nach Korpel (1995) entspricht (siehe auch Meyer et al 2003).

Ein so hoher Anteil an Beständen in der Zerfallsphase ist in produktiven Wäldern der Schweiz aus ökonomischer Sicht nicht sinnvoll. Dadurch würde eine nicht erwünschte Verknappung der natürlichen Ressource Holz erreicht. Auch wenn die zur Berechnung der Minimalfläche verwendeten Schwellenwerte des Totholzvolumens mit den mittleren Totholzvolumen von Naturwaldreservaten vergleichbar sind, ist es angesichts der hohen Flächenanteile der Zerfallsphase in Natur- und Urwäldern (Tabelle 3) jedoch fraglich, ob die angestrebte Fläche von 5\% für Altholzinseln und Naturwaldreservate reichen werden, um die xylobionte Diversität zu erhalten.
Den Altholzinseln kommt bei der Förderung xylobionter Arten eine wichtige Rolle zu. Im Optimalfall können sie geeignete Lebensräume zur Verfügung stellen, sind sie Nischen für Teilpopulationen und fungieren sie als vernetzende Trittsteine zwischen Naturwaldreservaten. Diesbezüglich stellt sich zwingend die Frage nach der optimalen Distanz zwischen Altholzinseln respektive zwischen Altholzinseln und Naturwaldreservaten, damit diese eine solche vernetzende Funktion übernehmen können. Da Antworten darauf noch weitgehend fehlen, sind weiterführende Forschungsarbeiten erforderlich. Dabei ist zu beachten, dass entsprechende Ergebnisse immer spezifisch für einzelne Arten oder Gruppen gelten. Deren sorgfältige Auswahl ist dementsprechend von grosser Bedeutung. Weiter möchten wir betonen, dass Altholzinseln kein Ersatz für Naturwaldreservate sind, da sie flächenmässig zu klein sind, um einen echten Prozessschutz zu gewährleisten. Vielmehr sollen Altholzinseln der Vernetzung von schon bestehenden Naturwaldreservaten und der ökologischen Aufwertung von Wirtschaftswäldern dienen.

\section{Fazit für die Umsetzung}

Das zunehmende Bewusstsein für die $\mathrm{CO}_{2}$-Problematik und die Verknappung der Erdölreserven führt zu einem erhöhten Interesse an Holz als natürlichem, nachwachsendem und regionalem Rohstoff. Es muss demzufolge angenommen werden, dass diese Entwicklungen eine Intensivierung der Holznutzung zur Folge haben werden. Die Akzeptanz grosser Waldreservate mit langfristigen Verträgen in gut zugänglichen Beständen ist aus diesem Grund nicht immer gewährleistet. Umso wichtiger ist die Möglichkeit, kleinere Flächen in Form von Altholzinseln auszuscheiden. Diese Projekte haben eine gute Chance auf Akzeptanz bei Waldeigentümern und Entscheidungsträgern, da sie die Nutzung nur auf verhältnismässig kleinen Flächen verhindern. In den momentan laufenden kantonalen Massnahmen zur Förderung der Biodiversität kommen Minimalflächen von 0.05 bis 2 ha zur Anwendung. Der Kanton Aargau zum Beispiel hat mit 2 ha die grösste Minimalfläche für Altholzinseln definiert. Er hat gleichzeitig mit rund 950 ha (Stand Dezember 2010) auch die schweizweit grösste Gesamtfläche an Altholzinseln ausgeschieden. Dieses Beispiel zeigt, dass die durch diese Studie geforderten Minimalflächen (0.7 bis 1.2 ha) im Jura und im Mittelland durchaus praktikabel sind. Auch international werden ähnliche Minimalflächen vorgeschlagen. Je-

\footnotetext{
1 BUWAL (2001) Leitsätze einer Waldreservatspolitik Schweiz. Bern: Bundesamt Umwelt Wald Landschaft. www.news.admin. ch/NSBSubscriber/message/attachments/4422.pdf (22.7.2011)
} 
dicke (2008) empfiehlt beispielsweise im Rahmen einer Arbeit zu Biotopverbunden für Alt- und Totholzlebensräume für Altholzinseln eine Minimalfläche von 1 bis 5 ha.

Mit den hier vorgeschlagenen Werten für die minimale Grösse von Altholzinseln kann sichergestellt werden, dass die Qualität der Altholzinseln bei der Ausscheidung mit hoher Wahrscheinlichkeit einem Mindestmass entspricht, welches die Förderung xylobionter Organismen ermöglicht. Durch eine nationale Vereinheitlichung der Minimalfläche wäre die Grundqualität der Altholzinseln gesichert, eine Voraussetzung für eine angemessene Abgeltung durch Bund und Kantone. Genauso wichtig wie die Minimalfläche ist aber auch, dass für die Altholzinseln die richtigen Bestände ausgewählt werden. So weisen Bestände, welche während längerer Zeit (mindestens 30 Jahre) nicht bewirtschaftet wurden und ein Alter von mindestens 120 Jahren haben, sehr gute Voraussetzungen auf (Bütler \& Lachat 2009).

Daneben ist auch die natürliche Heterogenität des Waldes zu beachten. So sollte darauf geachtet werden, dass Altholzinseln in Bereichen mit hohem Totholzanteil errichtet werden, damit ihre Qualität von Anfang an hoch ist. Zudem sollten die Bestände eine standortgemässe Baumartenzusammensetzung aufweisen, und Sicherheitsrisiken sind so weit als möglich zu minimieren. Bei der grossräumigen Planung von Altholzinseln sollte weiter darauf geachtet werden, dass verschiedene Waldtypen berücksichtigt und bestehende Naturwaldreservate möglichst vernetzt werden. So kann die Chance für eine Besiedlung der Altholzinseln durch xylobionte Arten verbessert werden, und bestehenden Populationen wird die Möglichkeit zur weiteren Ausbreitung gegeben.

Eingereicht: 2. Februar 2011, akzeptiert (mit Review): 11. Juli 2011

\section{Dank}

Diese Studie wurde vom Bundesamt für Umwelt finanziert. Besten Dank an Beat Wermelinger für seine Kommentare zu einer ersten Manuskriptfassung.

\section{Literatur}

ANDELMAN SJ, FAGAN WF (2000) Umbrellas and flagships: Efficient conservation surrogates or expensive mistakes? Proc Natl Acad Sci USA 97: 5954-5959.

ARRHENIUS O (1920) Species and area. J Ecol 9: 95-99.

BÖHL J, BRÄNDLI UB (2007) Deadwood volume assessment in the third Swiss National Forest Inventory: methods and first results. Eur J For Res 126: 449-457.

BRÄNDLI UB, ABEGG M (2009) Ergebnisse des Landesforstinventars LFI3. Der Schweizer Wald wird immer natürlicher. Wald Holz 90 (7): 27-29.
BÜTLER R, LACHAT T (2009) Wälder ohne Bewirtschaftung: eine Chance für die saproxylische Biodiversität. Schweiz Z Forstwes 160: 324-333. doi: 10.3188/szf.2009.0324

CHRISTENSEN M ET AL (2005) Dead wood in European beech (Fagus sylvatica) forest reserves. For Ecol Manage 210: 267-282.

DENNY MW, LUKE JH, HUNT LP, HARLEY CDG (2009) On the prediction of extreme ecological events. Ecol Monogr 79: 397-421.

DREVER MC, AITKEN KEH, NORRIS AR, MARTIN K (2008) Woodpeckers as reliable indicators of bird richness, forest health and harvest. Biol conserv 141: 624-634.

ELTON CS (1966) The pattern of animal communities. London: Methuen. 432 p.

GROVE S (2002) Saproxylic insect ecology and the sustainable management of forests. Ann Rev Ecol Syst 33: 1-23.

IRMLER U, ARP H, NOTZOLD R (2010) Species richness of saproxylic beetles in woodlands is affected by dispersion ability of species, age and stand size. J Ins Conserv 14: 227-235.

JAKOBY O, RADEMACHER C, GRIMM V (2010) Modelling dead wood islands in European beech forests: how much and how reliably would they provide dead wood? Eur J For Res 129: 659-668.

JEDICKE E (2008) Biotopverbund für Alt- und Totholzlebensräume. Leitlinien eines Schutzkonzeptes inner- und ausserhalb von Natura 2000. Nat.schutz Landsch.plan 40: 379-385.

KAUFMANN E (2000) Tarife für Schaftholz in Rinde und Rundholzsortimenten. Birmensdorf: Eidgenöss Forsch.anstalt Wald Schnee Landschaft. 53 p.

KORPEL S (1995) Die Urwälder der Westkarpaten. Stuttgart: Gustav Fischer. 310 p.

LEIBUNDGUT H (1982) Europäische Urwälder der Bergstufe. Bern: Haupt. 308 p.

LINDENMAYER D, MARGULES CR, BOTKIN D (2000) Indicators of biodiversity for ecologically sustainable forest management. Conserv Biol 14: 941-950.

MAYER H, NEUMANN M (1981) Struktureller und entwicklungsdynamischer Vergleich der Fichten-Tannen-Buchen-Urwälder Rothwald/Niederösterreich und Corkova Uvala/Kroatien. Forstwiss Cent.bl 100: 111-132.

MCPFE (2003) Improved Pan-European indicators for sustainable forest management. Vienna: Ministerial Conference on the Protection of Forests in Europe. $6 \mathrm{p}$.

MEYER P, TABAKU V, LÜPKE BV (2003) Die Struktur albanischer Rotbuchen-Urwälder - Ableitungen für eine naturnahe Buchenwirtschaft. Forstwiss Cent.bl 122: 47-58.

MEYER P, SCHMIDT M (2011) Accumulation of dead wood in abandoned beech (Fagus sylvatica) forest in northwestern Germany. For Ecol Manage 261: 342-352.

REMM J, LOHMUS A, REMM K (2006) Tree cavities in riverine forests: What determines their occurrence and use by hole-nesting passerines? For Ecol Manage 221: 267-277.

RICOTTA C (2009) Computing parametric beta diversity with unequal plot weigths: solution based on resampling methods. Theor Ecol 2: 13-17.

ROBERGE JM, ANGELSTAM P (2004) Usefulness of the umbrella species concept as a conservation tool. Conserv Biol 18: 76-85.

SEDDON PJ, LEECH T (2008) Conservation short cut, or long and winding road? A critique of umbrella species criteria. Oryx 42: 240-245.

SIITONEN J (2001) Forest management, coarse woody debris and saproxylic organisms: Fennoscandian boreal forests as an example. Ecol Bull 49: 11-41. 
SIITONEN J, MARTIKAINEN P, PUNTTILA P, RAUH J (2000) Coarse woody debris and stand characteristics in mature managed and old-growth boreal mesic forests in southern Finland. For Ecol Manage 128: 211-225.

TABAKU V (1999) Struktur von Buchen-Urwäldern in Albanien im Vergleich mit deutschen Buchen-Naturwaldreservaten und -Wirtschaftswäldern. Göttingen: Cuvillier. 206 p.
WINTER S (2008) Mikrohabitate und Phasenkartierung als Kern der Biodiversitätserfassung im Wald. Freising: Bayer Landesanstalt Wald Forstwirtschaft, LWF-Aktuell 63: 40-42.

WINTER S, MÖLLER GC (2008) Microhabitats in lowland beech forests as monitoring tool for nature conservation. For Ecol Manage 255: 1251-1261.

\section{Wie gross sollen Altholzinseln sein?}

Bis 2030 sollen etwa 5\% der Schweizer Waldfläche als Naturwaldreservate und Altholzinseln ausgewiesen sein. Dieses von Bund und Kantonen definierte Ziel soll die natürliche Waldentwicklung zulassen und die Vielfalt der holzbewohnenden Waldorganismen fördern. Neben den grossflächigen Naturwaldreservaten kommt auch den kleinflächigeren Altholzinseln eine bedeutende Rolle zu. Aufgrund von Stichprobenaufnahmen in seit mindestens 30 Jahren nicht mehr bewirtschafteten Hochwäldern wurde ein Modell zur Bestimmung der Minimalflächen von Altholzinseln erarbeitet. Zur Anwendung kamen dabei drei strukturbasierte Indikatoren (Gesamtzahl von Habitatstrukturen, Totholzvolumen und Anzahl Spechtbäume), anhand derer sich das ökologische Potenzial einer Altholzinsel ableiten lässt. Der Schwellenwert für das ökologische Potenzial wurde als Mittelwert des jeweiligen Indikators abzüglich des Standardfehlers definiert. Mithilfe der Bootstrapping-Methode wurde in der Folge die Flächengrösse modelliert, bei der $75 \%$ der Flächen den Schwellenwert übertrafen, also das ökologische Potenzial mit grosser Wahrscheinlichkeit erreicht wird. Diese Minimalfläche beträgt 0.7 ha für Auenwälder, 0.9 ha für Buchenwälder und TannenBuchen-Wälder und 1.2 ha für Tannen-Fichten-Wälder. Die berechneten Werte sollen als Minimalwerte für Altholzinseln und nicht als optimale Flächengrössen verstanden werden, da mit zunehmender Grösse die Langlebigkeit sowie Quantität und Qualität der Habitate ansteigen. Wenn die Umstände es zulassen, sind daher grössere Altholzinseln zu bevorzugen.

\section{Quelle est la surface minimale pour des îlots de sénescence?}

D'ici à 2030,5\% de la surface de la forêt suisse devrait être constituée de réserves forestières naturelles et d'îlots de sénescence. Cet objectif formulé par la Confédération et les cantons a pour but de favoriser le développement naturel des forêts et de conserver la diversité des espèces saproxyliques. En plus des grandes réserves forestières, les îlots de sénescence de plus petite taille jouent un rôle important.

Un modèle basé sur des observations de terrain réalisées dans des hautes futaies non exploitées depuis au moins 30 ans a été conçu pour déterminer la surface minimale des îlots de sénescence. Le potentiel écologique des îlots de sénescence a été évalué en se basant sur divers indicateurs de structures d'habitat (nombre total de structures d'habitat, volume de bois mort, nombre d'arbres à cavité de pic). Le seuil pour le potentiel écologique correspond à la moyenne de chaque indicateur moins l'erreur standard. Une surface minimale pour les îlots de sénescence de quatre types de forêt a été déterminée au moyen d'une méthode de rééchantillonnage lorsque $75 \%$ des cas dépassent les valeurs seuils: 0.7 ha pour la forêt alluviale, 0.9 ha pour la hêtraie, 0.9 ha pour la hêtraie à sapins et 1.2 ha en pessière-sapinière. Les surfaces calculées ne doivent pas être considérées comme des valeurs optimales, car la durabilité ainsi que les qualité et quantité des structures d'habitat augmentent avec un accroissement de la surface. Lorsque les circonstances le permettent, des îlots de sénescence de plus grande taille sont à envisager. 